\title{
HotSHOT Plus ThermalSHOCK, a new and efficient technique for preparation of PCR-quality mite genomic DNA
}

\author{
S. Alasaad \& L. Rossi \& S. Maione \& S. Sartore \& \\ R. C. Soriguer \& J. M. Pérez \& R. Rasero \& X. Q. Zhu \& \\ D. Soglia
}

\begin{abstract}
The present study adapted the HotSHOT method, a technique which has been successfully applied on different kinds of tissues, to studies of Sarcoptes. Some modifications of this technique were made which allowed the quick preparation of PCR-quality Sarcoptes genomic DNA (gDNA), namely applying sodium hydroxide as a substrate for three cycles of thermal shock, followed by a short incubation and $\mathrm{pH}$ adjustment with a Tris solution (HotSHOT Plus ThermalSHOCK). The performance of this technique was tested by amplifying a 450-bp rDNA
\end{abstract}

S. Alasaad · J. M. Pérez

Departamento de Biología Animal, Biología Vegetal y Ecología,

Universidad de Jaén,

Campus Las Lagunillas, s. n.,

23071 Jaén, Spain

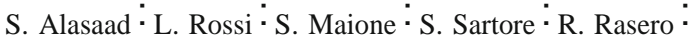

D. Soglia $(\star)$

Dipartimento di Produzioni Animali, Epidemiologia ed Ecologia,

Università di Torino,

Via Leonardo da Vinci 44,

10095 Grugliasco, Italy

e-mail: dominga.soglia@unito.it

R. C. Soriguer

Estación Biológica de Doñana,

Consejo Superior de Investigaciones Cientificas (CSIC),

Apartado 1056,

41080 Sevilla, Spain

X. Q. Zhu $(*)$

College of Veterinary Medicine,

South China Agricultural University,

483 Wushan Street, Tianhe District,

Guangzhou, Guangdong Province 510642,

People's Republic of China

e-mail: xingquanzh@scau.edu.cn fragment of the second internal transcribed spacer (ITS-2) and by multi-locus genotyping using ten microsatellites on 520 individual Sarcoptes samples. No difference in performance was observed between gDNA samples prepared using the HotSHOT Plus ThermalSHOCK technique and those prepared using a commercial kit utilizing proteinase $\mathrm{K}$ digestion. The results demonstrated that the HotSHOT Plus ThermalSHOCK technique is time-saving, economic, and easily automatable for the preparation of PCR-quality mite gDNA, which has implications for studying the molecular biology of mites with human and animal health significance. Although tested in the present study using Sarcoptes mites as a model, this technique may find broad applicability in extraction of gDNA from other parasites with small sizes and hard bodies.

Studies on the scabies agent, Sarcoptes scabiei, has indeed taken a leap into the molecular era, and the recent molecular advances have shown the potential for development of novel strategies towards the prevention, diagnosis, treatment, control, and immunotherapy of this important but neglected parasite (Walton et al. 2004). Nevertheless, due to the nature of S. scabiei, namely the small size (Fain 1978) and the difficulty to disrupt this hard-bodied parasite (hard chitinous exoskeleton), preparation of mite genomic DNA (gDNA) is generally known to be expensive (depending on commercial kits), laborious and time-consuming (requiring many steps including many hours of enzymatic digestion), with relatively high rate of failed reactions (Walton et al. 1997; Berrilli et al. 2002). The difficulties to obtain PCRquality Sarcoptes mite gDNA, together with the limited access to mite material (Mellanby 1944), make it necessary to find alternative methods or to adjust existing methods to the peculiarity of Sarcoptes genetic studies. 


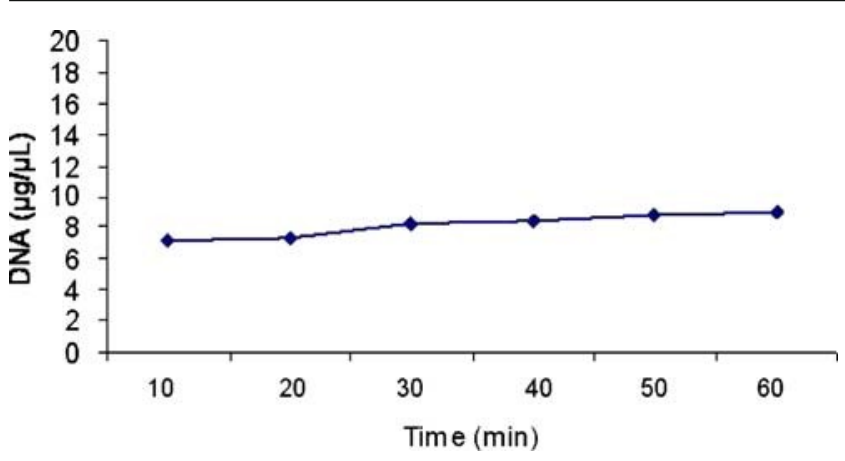

Fig. 1 Effect of heating time on the yield of soluble Sarcoptes mite genomic DNA prepared by the HotSHOT Plus ThermalSHOCK technique. DNA was prepared by three cycles of Thermal Shock $\left(-80^{\circ} \mathrm{C}\right.$ for $3 \mathrm{~min}$ and $+70^{\circ} \mathrm{C}$ for $\left.15 \mathrm{~s}\right)$ in $25 \mu \mathrm{l}$ alkaline lysis reagent, then heated for $10,20,30,40,50$, or $60 \mathrm{~min}$, followed by the addition of $25 \mu \mathrm{l}$ neutralizing reagent

Since its first application, HotSHOT has proven a rapid and inexpensive method to obtain good quality DNA from different tissue types (Truett et al. 2000; Jørgensen et al. 2005; Klover and Hennighausen 2007). The savings in time and reagents over traditional DNA preparation methods are substantial, and the quality of the gDNA is as good or slightly better than gDNA prepared by traditional methods. Based on known advantages of HotSHOT, the objective of the present study was to modify the original HotSHOT technique, by combination of some cycles of thermal shock (HotSHOT Plus ThermalSHOCK) to obtain PCR-quality Sarcoptes gDNA.

Five hundred and twenty adult mites were isolated from the skin of naturally infected European wild mammals, namely Alpine chamois Rupicapra rupicapra, red fox Vulpes vulpes, Alpine ibex Capra ibex and European moufflon Ovis aries musimon from Italy, as well as Southern chamois Rupicapra pyrenaica, Iberian ibex Capra pyrenaica and red fox from Spain. Mites were identified as Sarcoptes scabiei according to the morphological criteria of Fain (1968).

The reagents for HotSHOT Plus ThermalSHOCK were prepared as described previously (Truett et al. 2000) with some modifications, justified by the small size and the hard chitinous exoskeleton of S. scabiei. An alkaline lysis reagent with $25 \mathrm{mM} \mathrm{NaOH}, 0.2 \mathrm{mM}$ disodium EDTA and a $\mathrm{pH}$ of 12 was prepared by dissolving the salts in distilled water without adjusting the $\mathrm{pH}$. A neutralizing reagent with $40 \mathrm{mM}$ Tris- $\mathrm{HCl}$ and a $\mathrm{pH}$ of 5 was prepared by dissolving Tris- $\mathrm{HCl}$ in distilled water without adjusting the $\mathrm{pH}$. Two hundred and sixty Sarcoptes mites were placed individually into 96-well thermal cycler plates. Twenty-five microliters of alkaline lysis reagent was added to each well, and followed by three cycles of thermal shock (aiming to disrupt the exoskeleton) consisting of $2 \mathrm{~min}$ at $-80^{\circ} \mathrm{C}$ (freezing step) and $15 \mathrm{~s}$ at $+70^{\circ} \mathrm{C}$ (thawing step), then the samples were heated to $95^{\circ} \mathrm{C}$ for $10,20,30,40,50$, or 60 min (43 mite samples for each temperature point). After heating, the samples were cooled to $4^{\circ} \mathrm{C}$ and $25 \mu \mathrm{l}$ neutralizing reagent were added to each sample. The resulting buffer from the combination of the two reagents consisted of $20 \mathrm{mM}$ Tris-HCl (pH 8.1) and $0.1 \mathrm{mM}$ EDTA, which was similar to the traditional DNA storage buffer.

For comparative purpose, the commercial NucleoSpin Tissue Kit (Macherey-Nagel, Düren, Germany) using proteinase $\mathrm{K}$ digestion with one step of overnight lysis was used to obtain PCR-quality mite gDNA from 260 individual Sarcoptes mites according to the manufacturer's recommendations. The resulting volume of each sample was $50 \mu \mathrm{l}$, the same as that in HotSHOT Plus ThermalSHOCK technique.

The performance of gDNA samples extracted by the HotSHOT Plus ThermalSHOCK technique and those prepared using the commercial kit was tested and compared by amplifying the second internal transcribed spacer (ITS2) of ribosomal DNA (rDNA), as well as by multi-locus

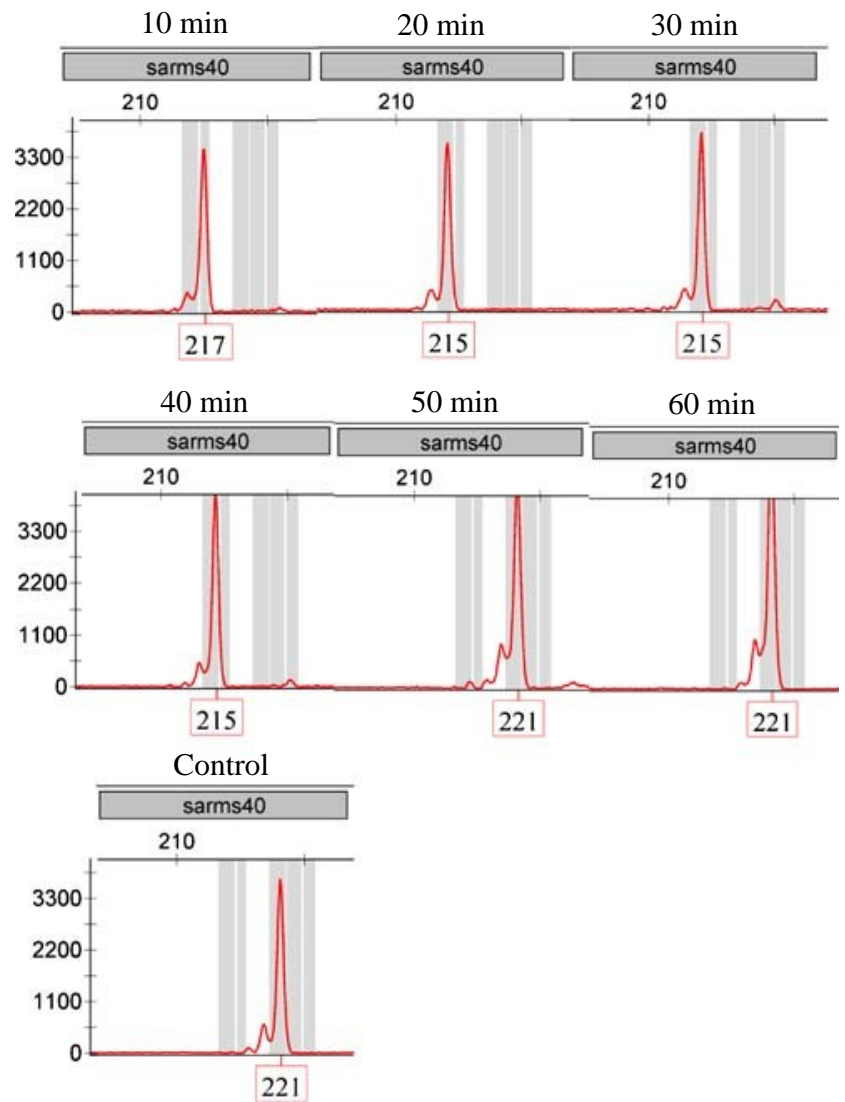

Fig. 2 Representative samples showing the effect of heating time on the amplification of Sarms-40 fluorescent-based microsatellite DNA from Sarcoptes scabiei. Control DNA was prepared using a commercial kit. HotSHOT plus ThermalSHOCK was performed as described in Fig. 1 
10× genotyping using microsatellite markers. The ITS-2 rDNA ( 450 bp) was amplified by PCR using primers RIB18 and RIB-3 as reported by Zahler et al. (1999) and following these authors' protocols. From the panel described by Walton et al. (1997), ten microsatellites (SARMS 33, 34, 35, 36, 37, 38, 40, 41, 44, and 45) were selected and applied with one multiplex 10× PCR as reported by Soglia et al. (2007), using Sarcoptes mite gDNA samples prepared by HotSHOT Plus ThermalSHOCK or those prepared using the commercial kit. The yield of soluble DNA was measured, only for successful reactions, by A260:A280 nm absorbance ratio using a spectrophotometer.

In the present study, 5,720 locus-specific reactions were carried out, and 773 reactions out of 2,860 (27.0\%) failed with gDNA prepared by HotSHOT Plus ThermalSHOCK, whereas 776 reactions out of 2,860 (27.1\%) failed with gDNA prepared by the traditional method. Evidence shows that no statistical difference exists between the two methods (Fisher's exact test) regarding the number of failed reactions. Nevertheless, the number of reactions failed by the HotSHOT Plus ThermalSHOCK varied with the heating time, being statistically lower when 10 min heating time was applied $(P<0.001)$, while no differences occurred following application of the remaining temperature points, which could be attributed to the hard chitinous exoskeleton of this parasite. The yield of the soluble DNA using the HotSHOT Plus ThermalSHOCK technique increased slightly with the increase of heating time (Fig. 1). However, the variation in soluble DNA among different durations of heating time had little influence on the amplification of the PCR product (Fig. 2).

The findings are consistent with the results obtained by Truett et al. (2000), applying HotSHOT technique on different tissue types. The results of the present study unambiguously show that mite gDNA prepared by HotSHOT Plus ThermalSHOCK is as good as the Sarcoptes gDNA prepared using the commercial kit and that the duration of heating time is not critical to the reaction outcomes and it may be chosen for the convenience of investigators, between 20 and $60 \mathrm{~min}$.

In conclusion, the HotSHOT Plus ThermalSHOCK technique was applied successfully to obtain PCR-quality Sarcoptes mite gDNA from hundreds of single Sarcoptes samples with no statistical differences compared with the commercial kit. The HotSHOT Plus ThermalSHOCK technique is time-saving, economic, and easily automatable for the preparation of PCR-quality mite gDNA, which has implications for studying the molecular biology of mites with human and animal health significance. Although evaluated in the present study using Sarcoptes mites as a model, this technique may find broad applicability in extraction of gDNA from other parasites with small sizes and hard bodies.

Acknowledgements Project support was provided by MURST contract year 2004, Prot. 2004078701-001 to LR. We thank all the people who supported the Department of Animal Production, Epidemiology, and Ecology, University of Turin-Italy, and the RNM118 investigation group. Junta de Andalucía-Spain is thanked for supporting SA's investigation stay in Italy. XQZ is supported by a grant from the Program for Changjiang Scholars and Innovative Research Team in University (Grant No. IRT0723). The experiments comply with the current laws of the countries in which the experiments were performed.

\section{References}

Berrilli F, D’Amelio S, Rossi L (2002) Ribosomal and mitochondrial DNA sequence variation in Sarcoptes mites from different hosts and geographical regions. Parasitol Res 88:772-777

Fain A (1968) Étude de la variabilité de Sarcoptes scabiei avec une revisiondes Sarcoptidae. Acta Zool Pathol Antverp 47:1-196

Fain A (1978) Epidemiological problems of scabies. Int J Dermatol $17: 20-30$

Jørgensen HBH, Hansen MM, Bekkevold D, Ruzzante DE, Loeschcke V (2005) Marine landscapes and population genetic structure of herring (Clupea harengus L.) in the Baltic Sea. Mol Ecol 14:3219-3234

Klover P, Hennighausen L (2007) Postnatal body growth is dependent on the transcription factors signal transducers and activators of transcription 5a/b in muscle: A role for autocrine/paracrine insulin-like growth factor I. Endocrinology 148:1489-1497

Mellanby K (1944) The development of symptoms, parasitic infection and immunity in human scabies. Parasitology 35:197-206

Soglia D, Rasero R, Rossi L, Sartore S, Sacchi P, Maione S (2007) Microsatellites as markers for comparison among different populations of Sarcoptes scabiei. It J Anim Sci 7:214-216

Truett GE, Heeger P, Mynatt RL, Truett AA, Walker JA, Warman ML (2000) Preparation of PCR quality mouse genomic DNA with hot sodium hydroxide and Tris (HotSHOT). BioTechniques. 29:52-54

Walton SF, Currie BJ, Kemp DJ (1997) A DNA fingerprinting system for the ectoparasite Sarcoptes scabiei. Mol Biochem Parasitol 85:187-196

Walton SF, Holt DC, Currie BJ, Kemp DJ (2004) Scabies: new future for a neglected disease. Adv Parasitol 57:309-376

Zahler M, Essig A, Gothe R, Rinder H (1999) Molecular analyses suggest monospecificity of the genus Sarcoptes (Acari: Sarcoptidae). Int J Parasitol 29:759-766 